\title{
Elaboration De La Carte Géotechnique De La Ville De Lubumbashi Guide Technique De Sélection Des Sites D’implantation D'ouvrages Du Génie Civil
}

\author{
Kasongo wa Mutombo Portance, MSc \\ Université de Lubumbashi, Faculté des Sciences, Département de Géologie, \\ Lubumbashi, République Démocratique du Congo \\ Mukoko Kalenda Gustave, PhD \\ Université de Lubumbashi, Département de Génie Civil, Unité de Recherche \\ Structures et GéotechniqueLubumbashi, République Démocratique du \\ Congo \\ Kipata Mwabanwa Louis, PhD \\ Lunda Ilunga Jean Marie, PhD \\ Université de Lubumbashi, Faculté des Sciences, Département de Géologie, \\ Lubumbashi, République Démocratique du Congo
}

Doi: 10.19044/esj.2018.v14n36p407 URL:http://dx.doi.org/10.19044/esj.2018.v14n36p407

\begin{abstract}
Geotechnical data processing that ignores the spatial aspect leads to loss of information, specification errors, non-convergent and ineffective estimation, and prediction errors. These data often present a spatial dependence (problem of spatial autocorrelation) and a heterogeneity in space (clustering problem) and sometimes temporal. The geotechnical mapping of Lubumbashi soils based on 1672 observations according to the AASHTO classification system (designation ASTM D-3282 M145), divided these soils into 11 subgroups: A-1-a (sandy gravels with clays), A-1-b (gravelly sands with clays), A-2-4 (gravelly sands with silts), A-2-5 (sandy gravels plastic silts), A-2-6 (gravelly sands with clays), A-2-7 (gravelly sands with active clays), A-4 (silts with sands), A-5 (plastic silts with sands), A-6 (clays with sand), A-7-5 (active clays), A-7-6 (active clays). Class A-3 has not been identified. Soils with an index 5 contain clays of the illite type whereas the others consist of kaolinites on plasticity Casagrande chart. Class A-1 and A-2 are good for foundation engeneering and form the potential aquifers of the city (groundwater, drainage zone, groundwater recharge zone). They also constitute favorable sites for the implantation of the cimeteries (aerated soils) because the time necessary for the destruction of bodies is brief. In this case the rotation of 5 years can be used without problem for a city with dense
\end{abstract}


population like the city of Lubumbashi. Soils A-4 and A-5 are also good for subgrade-foundations, but are highly vulnerable to erosion and liquefaction. Soils A-6 and A-7 form impervious bedrock substrates. These sites can be used as the landfill site. They are also considered clay deposits in the manufacture of ceramics and refractory products. Taking into account the nature of the source rocks, the same lithology can give several types of soils depending on the parameters influencing weathering: climate (Lubumbashi's tropical climate), $\mathrm{pH}, \mathrm{Eh}$, topography, anthropization, etc. One type of soil may also come from several rock formations. The geotechnical map developed compared to the geological map of Lubumbashi, shows that soils A-1 and A2 come from conglomerates, sandstones and the lateritization process of rock formations very rich in iron such as dolomitic shale and shale. Soils A-4 and A-5 are often alteration products of silty shales and siltstones, while soils A-6 and A-7 are derived from the alteration of clay and dolomitic rocks: shales, dolomitic shales, silty shales, limestones and dolomites. The geological map is fondamental but not sufficient document for geotechnical studies. A detailed study of the soils and the state of weathering of the different parts of a rock mass is inevitable.

Keywords: AASHTO classification, Voronoi polygon, Geotechnical information system, Clustering, Digital Elevation Model

\section{Resume}

Le traitement des données géotechniques qui ignore l'aspect spatial conduit à une perte d'informations, à des erreurs de spécifications, à des estimations non convergentes et non efficaces, à des erreurs de prédiction. Ces données présentent souvent une dépendance spatiale (problème d'autocorrélation spatiale) et une hétérogénéité dans l'espace (problème de clustering) et parfois temporelle. La cartographie géotechnique des sols de Lubumbashi effectuée sur base de 1672 observations suivant la classification AASHTO (désignation ASTM D-3282 M145), réparti ces sols en 11 sousgroupes : A-1-a (graviers sablonneux à argiles), A-1-b (sables graveleux à argiles), A-2-4 (sables graveleux à limons), A-2-5 (graviers sablonneux à limons plastiques), A-2-6 (sables graveleux à argiles), A-2-7 (sables graveleux à argiles actives), A-4 (limons à sables), A-5 (limons plastiques à sables), A6 (argiles à sables), A-7-5 (argiles actives à sables), A-7-6 (argiles actives à sables). La classe A-3 n'a pas été identifiée à Lubumbashi. Les sols à indice 5 contiennent des argiles de type illites tandisque les autres sont constitués des kaolinites sur l'abaque de plasticité de Casagrande. Les sols de classe A-1 et A-2, constituent des bons supports de fondations d'ouvrages et forment des aquifères potentiels de la ville de Lubumbashi (nappe d'eau souterraine, zone de drainage, zone d'alimentation des nappes). Ils constituent également des 
sites favorables à l'implantation des cimétières (sols aérés) car le délai nécessaire à la destruction de corps est bref. Dans ce cas la rotation de 5 ans peut être utilisée sans problème pour une ville à population dense comme la ville de Lubumbashi. Les sols A-4 et A-5 constituent également des bons sousbassement des fondations, mais sont très vulnérables à l'érosion hydrique et à la liquefaction. Les sols A-6 et A-7 forment des substratums imperméables des aquifères. Ces sites peuvent servir à la mise en place des centres d'enfouissement technique. Ils sont également considérés comme gisement d'argiles dans la fabrication des ceramiques et des produits refractaires. En tenant compte de la nature des roches mères, une même lithologie peut donner plusieurs types des sols en fonction des paramètres qui influencent l'altération: le climat (climat tropical à Lubumbashi), le pH, le Eh, la topographie, l'anthropisation, etc. Un type des sols peut provenir également des plusieurs formations rocheuses. Ceci est plus remarquable dans les roches sédimentaires qui sont plus hétérogènes que les roches magmatiques et métamorphiques. La carte géotechnique élaborée comparée à la carte géologique de Lubumbashi, montre que les sols A-1 et A-2 proviennent des conglomérats, des grès et du processus de latéritisation des formations rocheuses très riches en fer comme les shales et les shales dolomitiques. Les sols A-4 et A-5 sont souvent des produits d'altération des shales gréseux et des microgrès tandis que les sols A-6 et A-7 proviennent de l'altération des roches argileuses et dolomitiques: les shales, les shales dolomitiques, les shales gréseux, les calcaires et les dolomies. La carte géologique est un document nécessaire mais non suffisante pour les études géotechniques. Une étude détaillée des sols et l'état d'altération des différentes parties d'un massif rocheux est inévitable.

Mots-clés: Classification AASHTO, polygone de Voronoï, Système d'informations géotechnique, Clustering, Modèle Numérique de Terrain

\section{Introduction}

L'augmentation de la densité de la population s'accompagne des nombreux problèmes géotechniques à résoudre :

- La recherche des terres arables pour l'agriculture;

- La recherche des terrains pouvant supporter des grands ouvrages du génie civil comme les fondations des buildings et des barrages. Ceci consiste à cartographier des terrains à portance élevée et à compressibilité faible ;

- La recherche des zones d'alimentation des aquifères pour fixer des périmètres de protection à proximité des captages d'eau souterraines ;

- La recherche des nouveaux sites d'implantation des centres 
d'enfouissement technique et des cimetières.

Ces problèmes et d'autres non cités, nécessitent une connaissance géotechnique des matériaux constitutifs de sites ciblés. Les données considérées ont toujours une composante spatiale. Dans la modélisation, la considération de l'approche spatiale est souvent négligée, l'ouvrage est considéré comme ponctuel. Ceci cause beaucoup de préjudices surtout aux ouvrages linéaires comme des routes, des chemins de fer et des pistes d'atterrissage. Pour considérer la spatialité des variables, les ingénieurs se contentent seulement des méthodes de la statistique descriptive en dimensionnant soit avec des valeurs moyennes ou modales. Pour ce faire, c'est surtout l'hétérogénéité verticale qui est considérée et ils font faire des sondages.

Ce traitement des données géotechniques qui ignore l'aspect spatial conduit à une perte d'informations, à des erreurs de spécifications, à des estimations non convergentes et non efficaces, à des erreurs de prédiction. Ces données présentent souvent une dépendance spatiale (problème d'autocorrélation spatiale) et une hétérogénéité dans l'espace (problème de clustering) et parfois temporelle.

L'objectif principal de cet article est d'arriver à élaborer la carte géotechnique de la ville de Lubumbashi, car l'ancienne carte qui a été établie par Sonia et al. (2015) se basait seulement sur une trentaine d'observations au niveau de toutes la ville.

Les données qui ont servi à l'élaboration de la carte géotechnique viennent d'un système d'informations géotechnique de la ville de Lubumbashi mis en place par Kasongo (2017).

La ville de Lubumbashi se trouve dans la province du Haut-Katanga en République Démocratique du Congo. Elle est comprise entre les méridiens $27^{\circ} 10^{\prime}$ et $27^{\circ} 40^{\prime}$ de longitude Est et les parallèles $11^{\circ} 20^{\prime}$ et $12^{\circ}$ de latitude Sud (figure 1). 


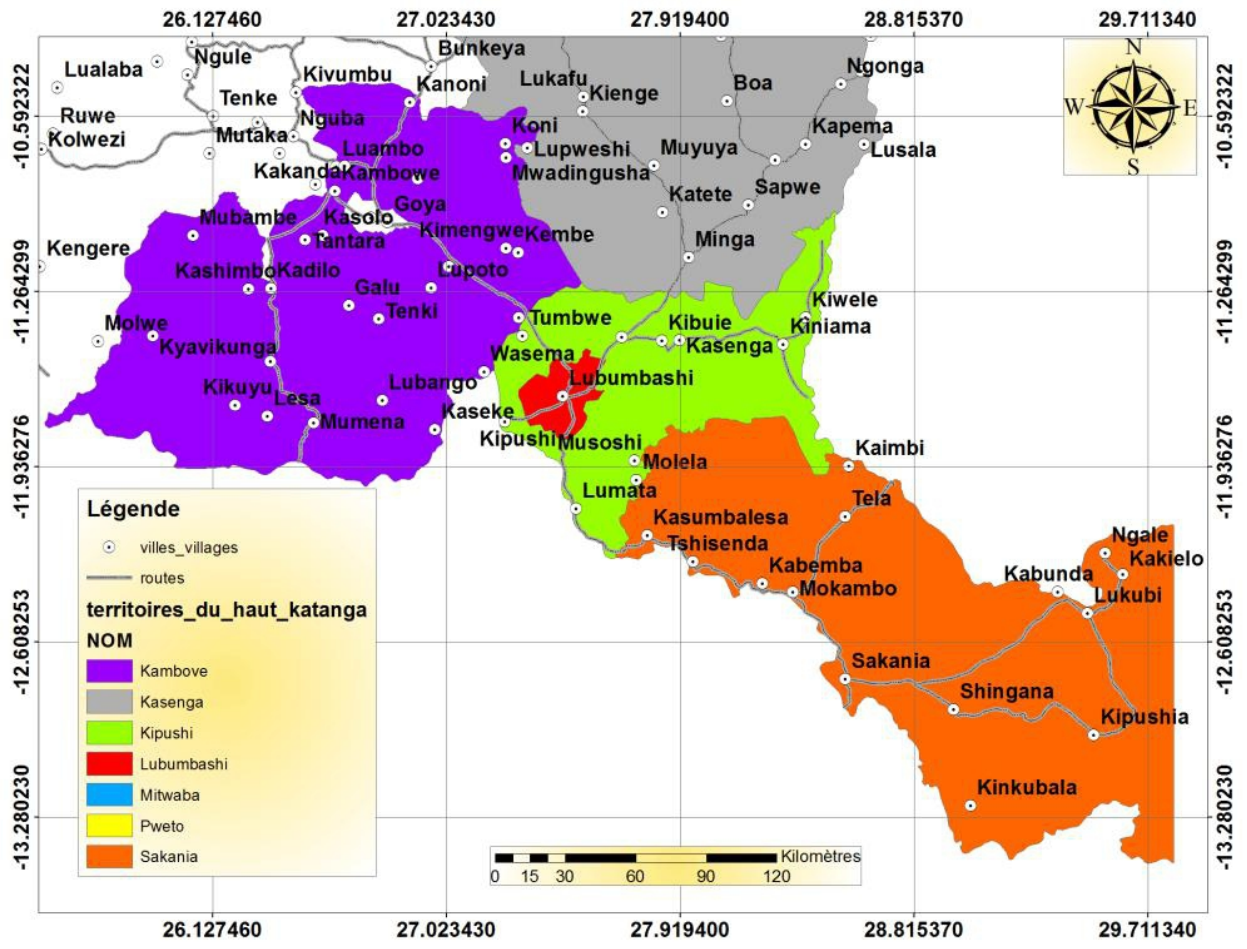

Figure 1: Localisation de la ville de Lubumbashi

Les méthodes d'analyse des données spatiales (cartographie numérique) ont été jugées efficaces pour répondre à la problématique de l'étude.

\section{Contexte géologique}

Les formations géologiques de Lubumbashi font parties du Katanguien. Le Katanguien est constitué d'une succession des formations sédimentaires mises en place entre l'orogenèse Kibarienne et l'orogenèse Lufilienne. Ce sont des sédiments d'une épaisseur d'environ $10 \mathrm{~km}$ et qui ont une grande extension partant de la Zambie jusqu'en République Démocratique du Congo.

Il est subdivisé en trois Groupes en se basant sur des données lithostratigraphiques avec comme repères deux niveaux tillitiques : Grand conglomérat et Petit conglomérat. Nous avons de bas en haut : le Roan, le Nguba et le Kundelungu.

Les travaux antérieurs montrent que sur l'ensemble de la ville et ses environs, on observe les formations géologiques suivantes : 


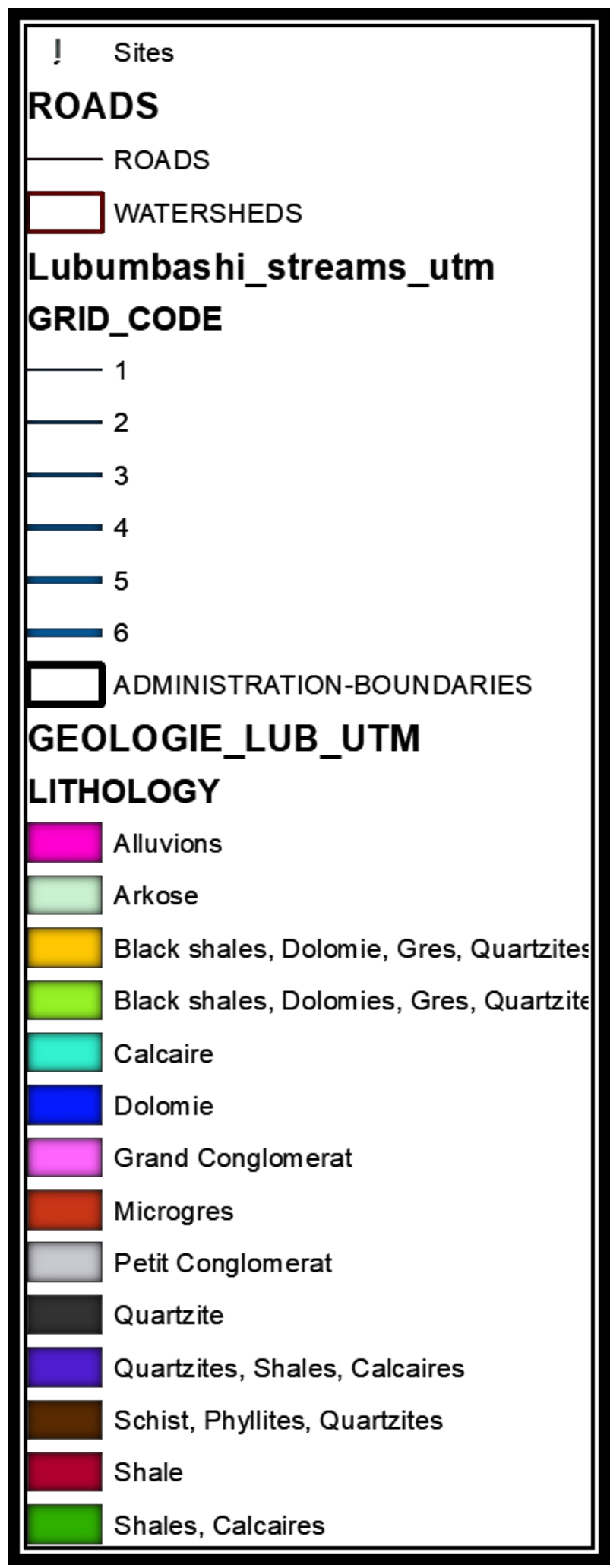


- Kundelungu indifférencié ;

- Alternance de microgrès assez dolomitiques en gros bancs et de shales peu dolomitiques à litage régulier. Ils appartiennent au Groupe de Kundelungu ;

- Dolomies grises, pourpre et beiges alternant avec des shales verts et gris (série récurrente), du sous-groupe de Monwezi ;

- La dolomie de Kakontwe : elle succède au Grand conglomérat et passe partiellement par des roches organogènes vers le Sud. Elle est formée par des roches détritiques. Elle appartient au groupe de Nguba ;

- Le Grand Conglomérat : sa puissance décroit du Nord vers le sud, avec trois niveaux distincts qui se marquent dans le relief par deux chaines de collines encadrant une dépression. On y trouve des intercalations non tillitiques variées : dolomies silicifiées (très rares), ou roches détritiques diverses (essentiellement des pélites vers le Sud, des pélites gréseuses au centre, etc.) ;

- Arkoses à grains fins ou grossiers, massives ou stratifiées ; elles appartiennent au sous-groupe de Mwashya ;

- Dolomies avec jaspes et oolites appartiennent au sous-groupe de Mwashya ;

- Shales, grès, dolomies et siltites : c'est une épaisse alternance de dolomies, grès, shales et qui appartiennent aux sous-groupes de Dipeta, de Mines et RAT Lilas.

Les affleurements sont rares dans la ville de Lubumbashi, et les plus importants sont ceux qu'on trouve dans la vallée de la rivière Lubumbashi et de part et d'autre du synclinal de Lubumbashi. Notons aussi que la série est normale à partir des coupes faites sur la ville de Lubumbashi. La figure 2 présente la carte géologique de Lubumbashi. Sa légende a été placée en flottant sur cette page pour sa visibilité. Les cours d'eau ont été classifiés suivant la classification de STRAHLER des thalwegs. 


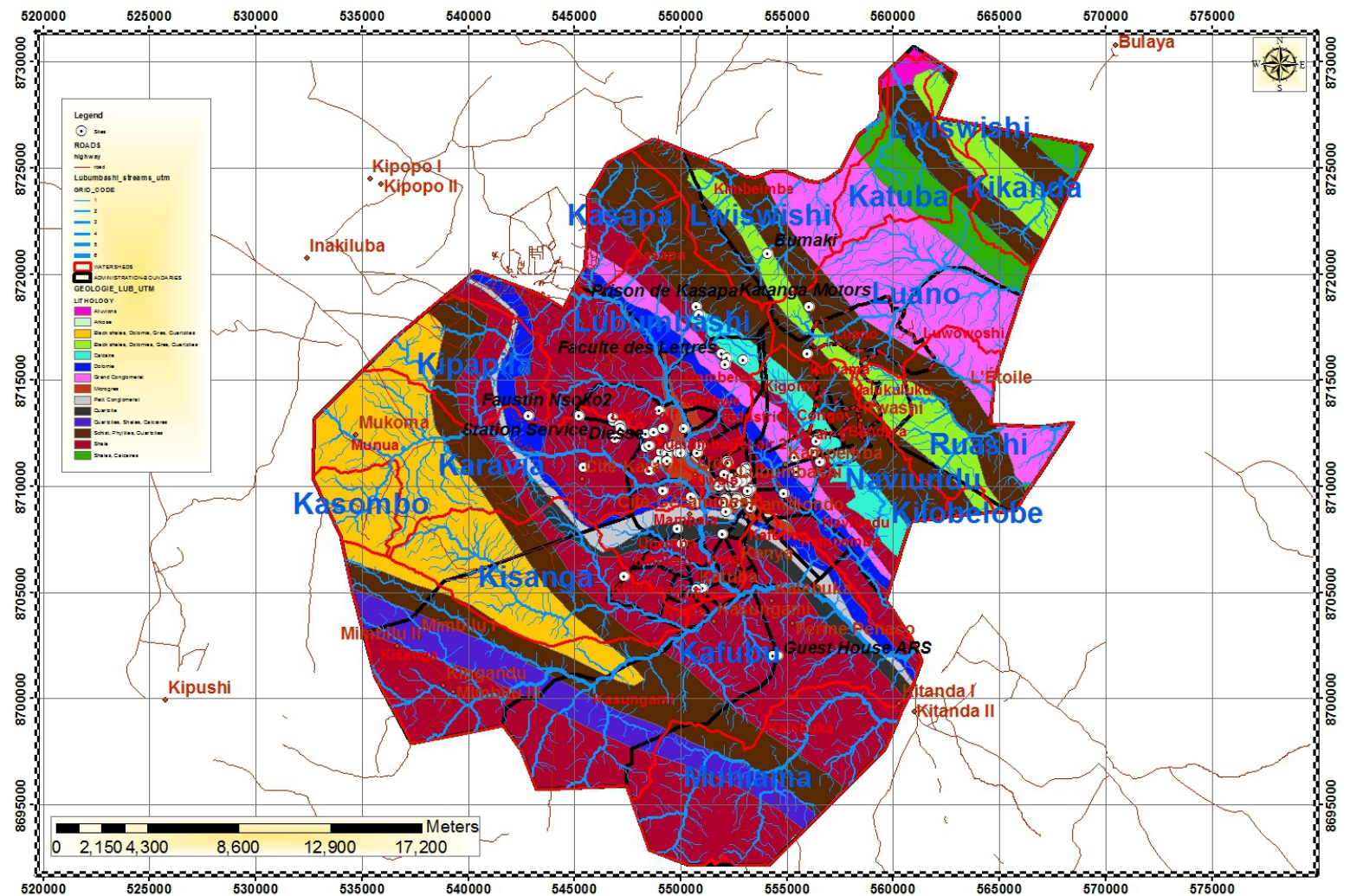

Figure 2 : Carte géologique de la ville de Lubumbashi

\section{Matériels et Méthodes}

Les données qui ont servi à l'établissement de la carte géotechnique ont été obtenues grâce aux essais suivants:

- l'analyse granulométrique suivant la norme NF P 94-056;

- essais des limites de consistance suivant la norme NF P 94-051.

Ces essais ont été réalisés suivant les normes géotechniques en vigueur (Verdeyen et al., 1968; Costet et al., 1975; De Clerck, 1977; Lousberg, 1978; Sanglerat et al., 1983; Dysli, 2001 ; Das et al. , 2012 ; Masamba, 2013). Les variables mesurées sont: la limite de liquidité $\omega_{l}$, la limite de plasticité $\omega_{p}$. A partir de l'analyse granulométrique nous avions déduit le passant au tamis de $80 \mu \mathrm{m}$, le passant au tamis de $400 \mu \mathrm{m}$ et le passant au tamis de $2 \mathrm{~mm} X_{2 \mathrm{~mm}}$. Ces variables ont servi à calculer d'autres qui en dérivent comme l'indice de plasticité $I_{p}$ et l'indice de groupe $I_{g}$.

Sur le terrain, les sols ont été décrits suivant la méthodologie géotechnique utilisée en Afrique du Sud pour éviter toutes subjectivités dans l'appréciation visuelle de l'état et de la nature des sols à échantillonner ( $A E G$ et al., 2001). La profondeur prospectée est en moyenne de $50 \mathrm{~cm}$. 
Après commutation de toutes les données dans notre système d'informations géotechniques (Kasongo, 2017), nous sommes arrivés à un échantillon de 1672 observations.

La classification géotechnique utilisée dans cet article est celle de AASHTO (American Association for State Highway Transportation Official) suivant la désignation ASTM D-3282 méthode AASHTO M145 (USDA, 1987; Das et al., 2012). D'autres classifications sont utilisées comme la classification USCS (Unified Soil Classification System). Ces classifications n'ont pas été retenues car les analyses granulométriques suivant la norme donnée, ne permettent pas d'obtenir la courbe granulométrique complète qui permettrait à déterminer le coefficient d'uniformité et de courbure dont dépendent souvent ces classifications.

Cette classification est complétée par la méthode des indices de groupe $I_{g}$ arrondi à l'unité suivant la formule de l'équation 1.

$I_{g}=\left(X_{200}-35\right)\left(0.2+0.005\left(\omega_{l}-40\right)\right)+0.01\left(X_{200}-15\right)\left(I_{p}-10\right)$

Où $X_{200}$ est le passant au tamis $N^{o} 200, \omega_{l}$ la limite de liquidité et $I_{p}$ l'indice de plasticité.

Pour le classe A-2-6 et A-2-7, on utilise la deuxième partie de l'équation $1\left(0.01\left(X_{200}-15\right)\left(I_{p}-10\right)\right)$. L'indice de groupe est le paramètre principal de clustering géotechnique.

Pour ressortir les données d'intérêt, nous avions recouru à la programmation orientée objet en utilisant le langage python (Laffitte, 1972; Dupre, 2010) et des bibliothèques tierces comme wxPython (Rappin et al., 2006) pour monter l'interface homme-machine; Scipy-Community (2015); Mckinney (2014) ; Hunter et al. (2016) pour l'analyse statistique des données. Ces méthodes ont été utilisées car des données qui sont à notre disposition sont massives (1672 enregistrements dans la base des données) et ne peuvent être traitées efficacement qu'avec des outils récents de fouille des données (Christopher et al., 2001 ;Lorenzo, 2006 ; Addinsoft, 2007; Edzer et al. 2008 ; Scipy-Community, 2015; Pysal-Developers, 2015). Les cartes géotechniques ont été tracées à l'aide du logiciel ARC GIS. A partir des polygones de Voronoï, nous avions achevé la carte géotechnique à l'aide des outils d'édition (union des polygones contigus présentant la même classe géotechnique).

Pour déterminer la nature des argiles contenues dans les sols identifiés, nous nous sommes servi de la classification basée sur l'abaque de Casagrande. Cette classification utilise l'indice de compression pour déduire la nature du minéral argileux dans le sol. Cet indice a été évalué à l'aide de la formule de Skempton (équation 2) à partir de la limite de liquidité des sols.

$C_{c}=0.009\left(\omega_{l}-10\right)$

La projection utilisée sur toutes nos cartes est WGS 84/UTM Zone 35 Sud (EPSG :32735). 
Les courbes de niveau et la délimitation des bassins versants des cours d'eau majeurs au niveau de la ville, ont été réalisées à l'aide du modèle numérique de terrain (MNT).

\section{Résultats et Discussion}

La classification utilisée dans cet article a ressortie les sous-groupe AASHTO suivants rangés selon leur ordre de comportement mécanique décroissant : A-1-a, A-1-b, A-2-4, A-2-5, A-2-6, A-2-7, A-4, A-5, A-6, A-75, A-7-6. Ces classes montrent des caractéristiques suivantes :

- Les sols de la classe A-1-a (tableau 1) sont représentés par des sols graveleux. Ils reposent sur les shales. Ces sols proviendraient du demantèlement des veines siliceuses qui affectent ces formations géologiques dans le secteur. Ils sont constitués de $52 \%$ de graviers (classe modale : graviers moyens), de $42 \%$ de sable (classe modale : sables gros), 6\% limons-argiles, une limite de liquidité moyenne de $28.24 \%$, un indice de plasticité moyen de $2.83 \%$, un indice de compression de 0.20 et un indice de vide moyen de 0.76 . Leur indice de groupe de 0 traduit que des chaussées peuvent être posées à même le sol;

Tableau 1 : Synthèse statistique des sols A-1-a

\begin{tabular}{|c|c|c|c|c|c|c|c|c|}
\hline & $\mathbf{N}$ & Moyenne & $\begin{array}{l}\text { Int. } \\
\text { Confiance } \\
-95 \%\end{array}$ & $\begin{array}{l}\text { Int. } \\
\text { Confiance } \\
+95 \%\end{array}$ & Q1 & Q3 & $\begin{array}{l}\text { Ecart- } \\
\text { type }\end{array}$ & $\begin{array}{l}\text { Coef. } \\
\text { Var. }\end{array}$ \\
\hline WI[\%] & 7 & 28.24 & 15.35 & 41.12 & 32.00 & 40.00 & 13.93 & 49.33 \\
\hline Ip[\%] & 7 & 2.83 & 0.83 & 4.83 & 3.50 & 5.15 & 2.17 & 76.57 \\
\hline Cc & 7 & 0.20 & 0.13 & 0.28 & 0.21 & 0.27 & 0.07 & 33.94 \\
\hline e & 7 & 0.76 & 0.45 & 1.07 & 0.85 & 0.96 & 0.33 & 43.65 \\
\hline X_4.75mm[\%] & 7 & 47.67 & 33.37 & 61.97 & 46.80 & 57.32 & 15.46 & 32.44 \\
\hline X_2mm[\%] & 7 & 15.31 & 10.82 & 19.81 & 14.74 & 20.41 & 4.86 & 31.75 \\
\hline$X \_400 \mu \mathrm{m}[\%]$ & 7 & 8.93 & 3.38 & 14.47 & 8.26 & 15.59 & 5.99 & 67.15 \\
\hline X_80 $\mu \mathrm{m}[\%]$ & 7 & 6.47 & 0.33 & 12.61 & 3.34 & 13.60 & 6.64 & 102.59 \\
\hline Ig & 7 & 0.00 & & & 0.00 & 0.00 & 0.00 & 0.00 \\
\hline GG[\%] & 7 & 0.00 & & & 0.00 & 0.00 & 0.00 & 0.00 \\
\hline GM[\%] & 7 & 50.99 & 37.25 & 64.72 & 52.87 & 56.50 & 14.85 & 29.12 \\
\hline GF[\%] & 7 & 1.34 & 0.00 & 4.63 & 0.00 & 0.00 & 3.56 & 264.58 \\
\hline SG[\%] & 7 & 34.39 & 20.16 & 48.62 & 36.61 & 44.92 & 15.39 & 44.75 \\
\hline SM[\%] & 7 & 4.93 & 0.17 & 9.69 & 3.75 & 6.43 & 5.14 & 104.33 \\
\hline $\mathrm{SF}[\%]$ & 7 & 2.64 & 0.00 & 5.32 & 1.34 & 5.64 & 2.90 & 109.81 \\
\hline $\mathbf{L} \_\mathbf{A}[\%]$ & 7 & 5.71 & 0.00 & 12.32 & 0.00 & 13.25 & 7.14 & 125.02 \\
\hline
\end{tabular}

- Les sols de la classe A-1-b (tableau 2) : proviennent de l'altération du Petit conglomérat, du Grand conglomérat, du complexe schistesphyllites-quartzites. Ils sont constitués de 32\% de graviers (classe modale : graviers moyens), de $52 \%$ de sable (classe modale : sables 
gros), $16 \%$ limons-argiles, une limite de liquidité moyenne de $20.57 \%$, un indice de plasticité moyen de $3.35 \%$, un indice de compression de 0.13 et un indice de vide moyen de 0.46 . Leur indice de groupe de 0 comme les A-1-a traduit que des chaussées peuvent être posées à même le sol ;

Tableau 2 : Synthèse statistique des sols A-1-b

\begin{tabular}{|l|l|l|l|l|l|l|l|l|}
\hline & $\mathbf{N}$ & Moyenne & $\begin{array}{l}\text { Int. } \\
\text { Confiance - } \\
\mathbf{9 5 \%}\end{array}$ & $\begin{array}{l}\text { Int. } \\
\text { Confiance } \\
\mathbf{+ 9 5 \%}\end{array}$ & $\mathbf{Q 1}$ & $\mathbf{Q 3}$ & $\begin{array}{l}\text { Ecart- } \\
\text { type }\end{array}$ & $\begin{array}{l}\text { Coef. } \\
\text { Var. }\end{array}$ \\
\hline WI[\%] & 6 & 20.57 & 9.47 & 31.67 & 20.77 & 27.73 & 10.57 & 51.41 \\
\hline Ip[\%] & 6 & 3.35 & 1.50 & 5.20 & 2.81 & 4.31 & 1.76 & 52.59 \\
\hline
\end{tabular}

Tableau 2 : Synthèse statistique des sols A-1-b (suite)

\begin{tabular}{|c|c|c|c|c|c|c|c|c|}
\hline & $\mathbf{N}$ & Moyenne & $\begin{array}{l}\text { Int. } \\
\text { Confiance } \\
-95 \%\end{array}$ & $\begin{array}{l}\text { Int. } \\
\text { Confiance } \\
+95 \%\end{array}$ & Q1 & Q3 & $\begin{array}{l}\text { Ecart- } \\
\text { type }\end{array}$ & $\begin{array}{l}\text { Coef. } \\
\text { Var. }\end{array}$ \\
\hline Cc & 6 & 0.13 & 0.09 & 0.17 & 0.11 & 0.16 & 0.03 & 24.41 \\
\hline e & 6 & 0.46 & 0.11 & 0.82 & 0.23 & 0.78 & 0.29 & 62.04 \\
\hline $\mathrm{X} \_4.75 \mathrm{~mm}[\%]$ & 6 & 62.00 & 44.87 & 79.13 & 49.60 & 71.62 & 16.32 & 26.33 \\
\hline X_2mm[\%] & 6 & 41.61 & 22.75 & 60.47 & 33.71 & 39.79 & 17.97 & 43.20 \\
\hline$X \_400 \mu m[\%]$ & 6 & 30.50 & 20.38 & 40.61 & 26.45 & 30.02 & 9.64 & 31.60 \\
\hline 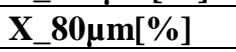 & 6 & 19.13 & 15.88 & 22.37 & 15.84 & 21.97 & 3.09 & 16.16 \\
\hline Ig & 6 & 0.00 & & & 0.00 & 0.00 & 0.00 & 0.00 \\
\hline GG[\%] & 6 & 1.28 & 0.00 & 4.58 & 0.00 & 0.00 & 3.14 & 244.95 \\
\hline GM[\%] & 6 & 29.88 & 14.90 & 44.85 & 28.38 & 32.55 & 14.27 & 47.75 \\
\hline GF[\%] & 6 & 1.02 & 0.00 & 2.69 & 0.00 & 2.81 & 1.59 & 155.71 \\
\hline SG[\%] & 6 & 32.25 & 19.41 & 45.09 & 28.80 & 38.07 & 12.23 & 37.94 \\
\hline SM[\%] & 6 & 7.92 & 1.25 & 14.60 & 3.62 & 11.88 & 6.36 & 80.29 \\
\hline $\mathrm{SF}[\%]$ & 6 & 11.22 & -0.49 & 22.92 & 5.35 & 12.57 & 11.15 & 99.46 \\
\hline L_A[\%] & 6 & 16.43 & 10.85 & 22.01 & 11.13 & 21.19 & 5.32 & 32.35 \\
\hline
\end{tabular}

- Les sols de la classe A-2-4 (tableau 3) : Ils sont générés par l'altération du Petit Conglomérat, de la dolomie, de quartzite, de shale gréseux. Ils sont constitués de $26 \%$ de graviers (classe modale: graviers moyens), de 58\% de sable (classe modale : sables gros), $16 \%$ limonsargiles, une limite de liquidité moyenne de $28.42 \%$, un indice de plasticité moyen de $7.48 \%$, un indice de compression de 0.17 et un indice de vide moyen de 0.79 . Leur indice de groupe de 0 comme les classes précédentes traduit que des chaussées peuvent être posées à même le sol ;

Tableau 3 : Synthèse statistique des sols A-2-4

\begin{tabular}{|l|l|l|l|l|l|l|l|l|}
\hline & $\mathbf{N}$ & Moyenne & $\begin{array}{l}\text { Int. } \\
\text { Confiance - } \\
\mathbf{9 5 \%}\end{array}$ & $\begin{array}{l}\text { Int. } \\
\text { Confiance } \\
\mathbf{+ 9 5 \%}\end{array}$ & $\mathbf{Q 1}$ & $\mathbf{Q 3}$ & $\begin{array}{l}\text { Ecart- } \\
\text { type }\end{array}$ & $\begin{array}{l}\text { Coef. } \\
\text { Var. }\end{array}$ \\
\hline WI[\%] & 55 & 28.42 & 26.65 & 30.20 & 28.00 & 32.20 & 6.57 & 23.13 \\
\hline Ip[\%] & 55 & 7.48 & 6.89 & 8.08 & 8.04 & 8.99 & 2.20 & 29.38 \\
\hline
\end{tabular}




\begin{tabular}{|c|c|c|c|c|c|c|c|c|}
\hline Cc & 54 & 0.17 & 0.16 & 0.18 & 0.16 & 0.20 & 0.05 & 28.73 \\
\hline e & 55 & 0.79 & 0.71 & 0.87 & 0.83 & 1.04 & 0.30 & 38.02 \\
\hline$X \_4.75 \mathrm{~mm}[\%]$ & 55 & 72.43 & 65.70 & 79.16 & 71.16 & 97.26 & 24.90 & 34.38 \\
\hline $\mathrm{X} \_2 \mathrm{~mm}[\%]$ & 55 & 48.68 & 41.24 & 56.12 & 41.34 & 85.13 & 27.51 & 56.52 \\
\hline$X \_400 \mu m[\%]$ & 55 & 35.38 & 30.71 & 40.04 & 33.33 & 53.53 & 17.24 & 48.74 \\
\hline 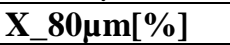 & 55 & 20.22 & 16.83 & 23.61 & 24.84 & 31.02 & 12.54 & 61.99 \\
\hline Ig & 55 & 0.00 & & & 0.00 & 0.00 & 0.00 & 0.00 \\
\hline GG[\%] & 55 & 0.74 & -0.38 & 1.85 & 0.00 & 0.00 & 4.12 & 559.52 \\
\hline GM[\%] & 55 & 18.68 & 14.35 & 23.00 & 22.60 & 33.16 & 15.99 & 85.63 \\
\hline GF $[\%]$ & 55 & 0.59 & -0.09 & 1.27 & 0.00 & 0.00 & 2.52 & 425.58 \\
\hline SG[\%] & 55 & 30.52 & 26.34 & 34.71 & 29.39 & 42.63 & 15.48 & 50.72 \\
\hline SM[\%] & 55 & 12.41 & 8.29 & 16.53 & 3.96 & 23.87 & 15.24 & 122.77 \\
\hline $\mathrm{SF}[\%]$ & 55 & 15.12 & 10.14 & 20.10 & 6.24 & 37.70 & 18.42 & 121.86 \\
\hline L_A[\%] & 55 & 16.00 & 12.43 & 19.56 & 16.78 & 29.24 & 13.19 & 82.46 \\
\hline
\end{tabular}

- Les sols de la classe A-2-5 (tableau 4) : Ils proviennent de l'altération des shales par latéritisation. Ils sont constitués de $46 \%$ de graviers (classe modale : graviers moyens), de $38 \%$ de sable (classe modale : sables gros), $16 \%$ limons-argiles, une limite de liquidité moyenne de $46.37 \%$, un indice de plasticité moyen de $6.69 \%$, un indice de compression de 0.33 et un indice de vide moyen de 0.95 . Leur indice de groupe de 0 comme les classes précédentes traduit que des chaussées peuvent être posées à même le sol;

Tableau 4 : Synthèse statistique des sols A-2-5

\begin{tabular}{|c|c|c|c|c|c|c|c|c|}
\hline & $\mathbf{N}$ & Moyenne & $\begin{array}{l}\text { Int. } \\
\text { Confiance - } \\
95 \%\end{array}$ & $\begin{array}{l}\text { Int. } \\
\text { Confiance } \\
+95 \%\end{array}$ & Q1 & Q3 & $\begin{array}{l}\text { Ecart- } \\
\text { type }\end{array}$ & $\begin{array}{l}\text { Coef. } \\
\text { Var. }\end{array}$ \\
\hline $\mathrm{WI}[\%]$ & 14 & 46.37 & 43.17 & 49.57 & 45.90 & 48.00 & 5.54 & 11.94 \\
\hline Ip[\%] & 14 & 6.09 & 4.60 & 7.58 & 6.65 & 8.00 & 2.58 & 42.35 \\
\hline $\mathrm{Cc}$ & 14 & 0.33 & 0.30 & 0.36 & 0.32 & 0.34 & 0.05 & 15.23 \\
\hline e & 14 & 0.95 & 0.87 & 1.04 & 0.99 & 1.04 & 0.15 & 15.56 \\
\hline$X \_4.75 \mathrm{~mm}[\%]$ & 14 & 54.10 & 37.19 & 71.01 & 64.21 & 78.18 & 29.28 & 54.12 \\
\hline $\mathrm{X} \_2 \mathrm{~mm}[\%]$ & 14 & 22.17 & 13.23 & 31.10 & 30.18 & 35.30 & 15.48 & 69.83 \\
\hline$X \_400 \mu m[\%]$ & 14 & 17.44 & 8.86 & 26.01 & 23.28 & 30.40 & 14.85 & 85.20 \\
\hline X_80um[\%] & 14 & 19.74 & 11.89 & 27.60 & 25.59 & 29.39 & 13.60 & 68.91 \\
\hline Ig & 14 & 0.00 & & & 0.00 & 0.00 & 0.00 & 0.00 \\
\hline GG[\%] & 14 & 0.00 & & & 0.00 & 0.00 & 0.00 & 0.00 \\
\hline GM[\%] & 14 & 29.62 & 17.30 & 41.94 & 27.05 & 38.06 & 21.34 & 72.05 \\
\hline GF $[\%]$ & 14 & 2.00 & -0.55 & 4.54 & 0.00 & 0.00 & 4.41 & 221.22 \\
\hline SG[\%] & 14 & 35.68 & 21.20 & 50.17 & 43.77 & 51.99 & 25.10 & 70.32 \\
\hline SM[\%] & 14 & 1.34 & 0.49 & 2.19 & 0.68 & 2.90 & 1.47 & 109.82 \\
\hline $\mathrm{SF}[\%]$ & 14 & 1.18 & 0.17 & 2.19 & 0.65 & 1.74 & 1.74 & 148.11 \\
\hline L_A[\%] & 14 & 15.90 & 7.50 & 24.31 & 20.66 & 28.73 & 14.56 & 91.55 \\
\hline
\end{tabular}


- Les sols de la classe A-2-6 (tableau 5) : Ils proviennent de l'altération du shale dolomitique, du complexe schistes-phyllites-quartzites, du Grand conglomérat. Ils sont constitués de $26 \%$ de graviers (classe modale : graviers moyens), de $62 \%$ de sable (classe modale : sables gros), $12 \%$ limons-argiles, une limite de liquidité moyenne de $31.92 \%$, un indice de plasticité moyen de $14.24 \%$, un indice de compression de 0.20 et un indice de vide moyen de 0.90 . Leur indice de groupe de 0 comme les classes précédentes traduit que des chaussées peuvent être posées à même le sol,

Tableau 5 : Synthèse statistique des sols A-2-6

\begin{tabular}{|c|c|c|c|c|c|c|c|c|}
\hline & $\mathbf{N}$ & Moyenne & $\begin{array}{l}\text { Int. } \\
\text { Confiance - } \\
95 \%\end{array}$ & $\begin{array}{l}\text { Int. } \\
\text { Confiance } \\
+95 \%\end{array}$ & Q1 & Q3 & $\begin{array}{l}\text { Ecart- } \\
\text { type }\end{array}$ & $\begin{array}{l}\text { Coef. } \\
\text { Var. }\end{array}$ \\
\hline $\mathrm{WI}[\%]$ & 64 & 31.92 & 30.77 & 33.07 & 32.00 & 36.00 & 4.61 & 14.43 \\
\hline Ip[\%] & 64 & 14.24 & 13.36 & 15.12 & 13.44 & 15.90 & 3.52 & 24.75 \\
\hline $\mathrm{Cc}$ & 64 & 0.20 & 0.19 & 0.21 & 0.20 & 0.23 & 0.04 & 21.01 \\
\hline e & 64 & 0.90 & 0.83 & 0.96 & 0.99 & 1.04 & 0.26 & 28.99 \\
\hline $\mathrm{X} \_4.75 \mathrm{~mm}[\%]$ & 64 & 62.07 & 54.39 & 69.74 & 61.94 & 84.87 & 30.73 & 49.50 \\
\hline $\mathrm{X} \_2 \mathrm{~mm}[\%]$ & 64 & 34.20 & 27.07 & 41.34 & 32.66 & 43.73 & 28.55 & 83.47 \\
\hline$X \_400 \mu m[\%]$ & 64 & 24.43 & 19.67 & 29.19 & 27.22 & 36.70 & 19.07 & 78.05 \\
\hline $\mathrm{X} \_80 \mu \mathrm{m}[\%]$ & 64 & 16.36 & 12.99 & 19.73 & 20.02 & 27.90 & 13.48 & 82.39 \\
\hline Ig & 64 & 0.00 & 0.00 & 0.00 & 0.00 & 0.00 & 0.00 & 0.00 \\
\hline GG[\%] & 64 & 0.12 & -0.12 & 0.36 & 0.00 & 0.00 & 0.96 & 800.00 \\
\hline GM[\%] & 64 & 22.01 & 17.35 & 26.67 & 23.81 & 38.06 & 18.65 & 84.75 \\
\hline GF $[\%]$ & 64 & 1.50 & 0.39 & 2.62 & 0.00 & 0.00 & 4.46 & 296.72 \\
\hline SG[\%] & 64 & 32.21 & 27.45 & 36.98 & 34.38 & 47.58 & 19.07 & 59.21 \\
\hline
\end{tabular}

Tableau 5 : Synthèse statistique des sols A-2-6 (suite)

\begin{tabular}{|l|l|l|l|l|l|l|l|l|}
\hline & $\mathbf{N}$ & Moyenne & $\begin{array}{l}\text { Int. } \\
\text { Confiance - } \\
\mathbf{9 5 \%}\end{array}$ & $\begin{array}{l}\text { Int. } \\
\text { Confiance } \\
\mathbf{+ 9 5 \%}\end{array}$ & $\mathbf{Q 1}$ & $\mathbf{Q 3}$ & $\begin{array}{l}\text { Ecart- } \\
\text { type }\end{array}$ & $\begin{array}{l}\text { Coef. } \\
\text { Var. }\end{array}$ \\
\hline SM[\%] & 64 & 8.64 & 4.98 & 12.29 & 2.53 & 5.02 & 14.62 & 169.33 \\
\hline SF[\%] & 64 & 9.18 & 5.90 & 12.46 & 2.92 & 7.69 & 13.15 & 143.25 \\
\hline L_A[\%] & 64 & 12.27 & 8.76 & 15.79 & 0.00 & 26.88 & 14.06 & 114.61 \\
\hline
\end{tabular}

- Les sols de la classe A-2-7 (tableau 6) : Ils sont générés par l'altération des calcaires, des shales. Ils sont constitués de 30\% de graviers (classe modale : graviers moyens), de $58 \%$ de sable (classe modale : sables gros), $12 \%$ limons-argiles, une limite de liquidité moyenne de $47.52 \%$, un indice de plasticité moyen de $20.05 \%$, un indice de compression de 0.34 et un indice de vide moyen de 0.98 . Leur indice de groupe de 0 comme les classes précédentes traduit que des chaussées peuvent être posées à même le sol ; 
Tableau 6 : Synthèse statistique des sols A-2-7

\begin{tabular}{|c|c|c|c|c|c|c|c|c|}
\hline & $\mathbf{N}$ & Moyenne & $\begin{array}{l}\text { Int. } \\
\text { Confiance - } \\
95 \%\end{array}$ & $\begin{array}{l}\text { Int. } \\
\text { Confiance } \\
+95 \%\end{array}$ & Q1 & Q3 & $\begin{array}{l}\text { Ecart- } \\
\text { type }\end{array}$ & $\begin{array}{l}\text { Coef. } \\
\text { Var. }\end{array}$ \\
\hline $\mathrm{WI}[\%]$ & 45 & 47.52 & 44.87 & 50.17 & 46.00 & 49.00 & 8.82 & 18.55 \\
\hline Ip[\%] & 45 & 20.05 & 16.93 & 23.17 & 16.94 & 22.80 & 10.37 & 51.73 \\
\hline $\mathrm{Cc}$ & 45 & 0.34 & 0.31 & 0.36 & 0.32 & 0.35 & 0.08 & 23.50 \\
\hline e & 45 & 0.98 & 0.93 & 1.04 & 1.04 & 1.04 & 0.18 & 17.81 \\
\hline X_4.75mm[\%] & 45 & 56.08 & 46.01 & 66.15 & 61.94 & 81.00 & 33.52 & 59.77 \\
\hline $\mathrm{X} \_2 \mathrm{~mm}[\%]$ & 45 & 28.22 & 19.64 & 36.80 & 26.40 & 39.79 & 28.55 & 101.18 \\
\hline$X \_400 \mu \mathrm{m}[\%]$ & 45 & 21.98 & 15.39 & 28.57 & 21.16 & 34.52 & 21.94 & 99.81 \\
\hline 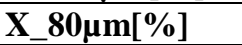 & 45 & 17.13 & 13.17 & 21.10 & 21.35 & 28.68 & 13.19 & 77.00 \\
\hline Ig & 45 & 0 & 0 & 0 & 0 & 0 & 0 & 0 \\
\hline GG[\%] & 45 & 0.54 & 0.00 & 1.63 & 0.00 & 0.00 & 3.63 & 670.82 \\
\hline GM[\%] & 45 & 21.74 & 15.59 & 27.88 & 21.24 & 38.06 & 20.45 & 94.10 \\
\hline GF [\%] & 45 & 0.55 & 0.00 & 1.16 & 0.00 & 0.00 & 2.05 & 375.06 \\
\hline SG[\%] & 45 & 30.17 & 23.41 & 36.93 & 26.11 & 48.73 & 22.50 & 74.57 \\
\hline SM[\%] & 45 & 6.86 & 2.91 & 10.81 & 0.89 & 3.29 & 13.14 & 191.53 \\
\hline SF [\%] & 45 & 6.89 & 3.13 & 10.65 & 1.74 & 3.39 & 12.52 & 181.67 \\
\hline L_A[\%] & 45 & 10.46 & 6.52 & 14.41 & 0.00 & 22.27 & 13.13 & 125.52 \\
\hline
\end{tabular}

- Les sols de la classe A-4 (tableau 7) : Ils proviennent du complexe schistes-phyllites-quartzites, des shales gréseux, des dolomies, des microgrès. Ils sont constitués de $3 \%$ de graviers, de $17 \%$ de sable, $80 \%$ limons-argiles, une limite de liquidité moyenne de $26.80 \%$, un indice de plasticité moyen de $5.48 \%$, un indice de compression de 0.16 et un indice de vide moyen de 1.07. Leur indice de groupe moyen est de 3;

Tableau 7 : Synthèse statistique des sols A-4

\begin{tabular}{|c|c|c|c|c|c|c|c|c|}
\hline & $\mathbf{N}$ & Moyenne & $\begin{array}{l}\text { Int. } \\
\text { Confiance } \\
-95 \%\end{array}$ & $\begin{array}{l}\text { Int. } \\
\text { Confiance } \\
+95 \%\end{array}$ & Q1 & Q3 & $\begin{array}{l}\text { Ecart- } \\
\text { type }\end{array}$ & $\begin{array}{l}\text { Coef. } \\
\text { Var. }\end{array}$ \\
\hline $\mathrm{WI}[\%]$ & 468 & 26.80 & 26.10 & 27.50 & 27.49 & 32.18 & 7.72 & 28.81 \\
\hline $\mathbf{I p}[\%]$ & 468 & 5.48 & 5.21 & 5.75 & 5.72 & 8.25 & 3.02 & 55.11 \\
\hline $\mathrm{Cc}$ & 456 & 0.16 & 0.15 & 0.16 & 0.16 & 0.20 & 0.06 & 37.04 \\
\hline e & 460 & 1.07 & 1.04 & 1.10 & 1.04 & 1.28 & 0.34 & 31.80 \\
\hline X_4.75mm[\%] & 468 & 93.55 & 91.92 & 95.19 & 100.00 & 100.00 & 17.95 & 19.19 \\
\hline
\end{tabular}

Tableau 7 : Synthèse statistique des sols A-4 (suite)

\begin{tabular}{|c|c|c|c|c|c|c|c|c|}
\hline & $\mathbf{N}$ & Moyenne & $\begin{array}{l}\text { Int. } \\
\text { Confiance } \\
-95 \%\end{array}$ & $\begin{array}{l}\text { Int. } \\
\text { Confiance } \\
+95 \%\end{array}$ & Q1 & Q3 & $\begin{array}{l}\text { Ecart- } \\
\text { type }\end{array}$ & $\begin{array}{l}\text { Coef. } \\
\text { Var. }\end{array}$ \\
\hline X_2mm[\%] & 468 & 87.66 & 85.57 & 89.76 & 99.46 & 100.00 & 23.04 & 26.28 \\
\hline$X \_400 \mu \mathrm{m}[\%]$ & 468 & 84.80 & 82.76 & 86.83 & 94.47 & 100.00 & 22.35 & 26.36 \\
\hline 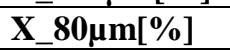 & 468 & 74.17 & 72.72 & 75.61 & 75.00 & 86.94 & 15.89 & 21.43 \\
\hline Ig & 468 & 2.89 & 2.63 & 3.15 & 2.00 & 5.00 & 2.85 & 98.69 \\
\hline GG[\%] & 468 & 0.00 & 0.00 & 0.00 & 0.00 & 0.00 & 0.00 & \\
\hline GM[\%] & 468 & 3.38 & 2.75 & 4.02 & 0.00 & 2.50 & 7.02 & 207.43 \\
\hline
\end{tabular}




\begin{tabular}{|l|l|l|l|l|l|l|l|l|} 
GF[\%] & 468 & 0.07 & 0.01 & 0.13 & 0.00 & 0.00 & 0.68 & 970.65 \\
\hline SG[\%] & 468 & 6.98 & 6.04 & 7.92 & 2.05 & 9.62 & 10.35 & 148.30 \\
\hline SM[\%] & 468 & 2.71 & 2.31 & 3.11 & 0.88 & 3.58 & 4.44 & 163.54 \\
\hline SF[\%] & 468 & 6.89 & 6.17 & 7.60 & 4.24 & 10.55 & 7.88 & 114.39 \\
\hline L_A[\%] & 468 & 54.75 & 51.57 & 57.93 & 66.76 & 83.58 & 35.01 & 63.94 \\
\hline
\end{tabular}

- Les sols de la classe A-5 (tableau 8): Ils proviennent du Grand conglomérat, de la dolomie. Ils sont constitués de $7 \%$ de graviers, de $18 \%$ de sable, $75 \%$ limons-argiles, une limite de liquidité moyenne de $45.05 \%$, un indice de plasticité moyen de $6.08 \%$, un indice de compression de 0.32 et un indice de vide moyen de 1.02 . Leur indice de groupe moyen est de 6 ;

Tableau 8 : Synthèse statistique des sols A-5

\begin{tabular}{|c|c|c|c|c|c|c|c|c|}
\hline & $\mathbf{N}$ & Moyenne & $\begin{array}{l}\text { Int. } \\
\text { Confiance - } \\
95 \%\end{array}$ & $\begin{array}{l}\text { Int. } \\
\text { Confiance } \\
+95 \%\end{array}$ & Q1 & Q3 & $\begin{array}{l}\text { Ecart- } \\
\text { type }\end{array}$ & $\begin{array}{l}\text { Coef. } \\
\text { Var. }\end{array}$ \\
\hline WI[\%] & 41 & 45.05 & 43.49 & 46.60 & 44.64 & 46.00 & 4.92 & 10.93 \\
\hline$I p[\%]$ & 41 & 6.08 & 5.16 & 7.00 & 6.87 & 8.30 & 2.92 & 48.08 \\
\hline Ce & 41 & 0.32 & 0.30 & 0.33 & 0.31 & 0.32 & 0.04 & 14.05 \\
\hline e & 41 & 1.02 & 0.94 & 1.10 & 1.04 & 1.07 & 0.26 & 24.93 \\
\hline $\mathbf{X} \_4.75 \mathrm{~mm}[\%]$ & 41 & 87.70 & 80.48 & 94.92 & 99.31 & 100.00 & 22.87 & 26.07 \\
\hline $\mathbf{X} \_2 \mathrm{~mm}[\%]$ & 41 & 78.93 & 70.37 & 87.48 & 96.94 & 100.00 & 27.11 & 34.36 \\
\hline $\mathrm{X}_{-400 \mu \mathrm{m}[\%]}$ & 41 & 75.62 & 66.77 & 84.47 & 90.66 & 99.53 & 28.04 & 37.08 \\
\hline $\mathrm{X} \_80 \mu \mathrm{m}[\%]$ & 41 & 70.81 & 64.28 & 77.34 & 68.12 & 92.86 & 20.69 & 29.21 \\
\hline Ig & 41 & 5.80 & 4.47 & 7.14 & 5.00 & 10.00 & 4.24 & 73.11 \\
\hline GG[\%] & 41 & 0.00 & & & 0.00 & 0.00 & 0.00 & 0.00 \\
\hline GM[\%] & 41 & 6.29 & 3.50 & 9.07 & 0.20 & 15.90 & 8.82 & 140.28 \\
\hline GF[\%] & 41 & 1.13 & 0.02 & 2.24 & 0.00 & 0.00 & 3.51 & 310.32 \\
\hline SG[\%] & 41 & 11.34 & 7.24 & 15.44 & 4.51 & 22.76 & 12.98 & 114.50 \\
\hline SM[\%] & 41 & 1.29 & 0.64 & 1.93 & 0.42 & 1.36 & 2.04 & 158.34 \\
\hline SF[\%] & 41 & 5.34 & 2.19 & 8.49 & 1.79 & 4.17 & 9.99 & 187.08 \\
\hline$L_{-} \mathbf{A}[\%]$ & 41 & 64.86 & 55.50 & 74.21 & 66.83 & 92.86 & 29.63 & 45.69 \\
\hline
\end{tabular}

- Les sols de la classe A-6 (tableau 9) : Ils proviennent de l'altération du shale, des calcaires, du Grand Conglomérat, du complexe schistesphyllites-quartzites, de la dolomie. Ils sont constitués de 3\% de graviers, de $17 \%$ de sable, $80 \%$ limons-argiles, une limite de liquidité moyenne de $33.02 \%$, un indice de plasticité moyen de $15.22 \%$, un indice de compression de 0.21 et un indice de vide moyen de 1.12. Leur indice de groupe moyen est de 10. Ce type de sols constitue la classe modale des sols de Lubumbashi ; 
Tableau 9 : Synthèse statistique des sols A-6

\begin{tabular}{|c|c|c|c|c|c|c|c|c|}
\hline & $\mathbf{N}$ & Moyenne & $\begin{array}{l}\text { Int. } \\
\text { Confiance } \\
-95 \% \\
\end{array}$ & $\begin{array}{l}\text { Int. } \\
\text { Confiance } \\
+95 \% \\
\end{array}$ & Q1 & Q3 & $\begin{array}{l}\text { Ecart- } \\
\text { type }\end{array}$ & $\begin{array}{l}\text { Coef. } \\
\text { Var. }\end{array}$ \\
\hline $\mathrm{WI}[\%]$ & 657 & 33.02 & 32.68 & 33.36 & 33.69 & 36.33 & 4.41 & 13.36 \\
\hline Ip[\%] & 657 & 15.22 & 14.93 & 15.51 & 14.60 & 17.05 & 3.81 & 25.04 \\
\hline $\mathrm{Cc}$ & 657 & 0.21 & 0.20 & 0.21 & 0.21 & 0.24 & 0.04 & 19.16 \\
\hline e & 657 & 1.12 & 1.09 & 1.15 & 1.07 & 1.31 & 0.39 & 34.65 \\
\hline $\mathrm{X} \_4.75 \mathrm{~mm}[\%]$ & 657 & 94.08 & 92.79 & 95.38 & 99.98 & 100.00 & 16.93 & 18.00 \\
\hline $\mathrm{X} \_2 \mathrm{~mm}[\%]$ & 657 & 88.47 & 86.80 & 90.14 & 99.17 & 100.00 & 21.84 & 24.69 \\
\hline 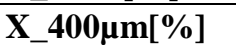 & 657 & 84.97 & 83.37 & 86.56 & 92.32 & 100.00 & 20.81 & 24.49 \\
\hline 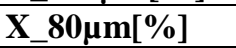 & 657 & 75.53 & 74.38 & 76.69 & 77.18 & 87.65 & 15.06 & 19.94 \\
\hline Ig & 657 & 9.86 & 9.53 & 10.18 & 10.00 & 13.00 & 4.25 & 43.15 \\
\hline GG[\%] & 657 & 0.01 & -0.01 & 0.03 & 0.00 & 0.00 & 0.21 & 1936.56 \\
\hline GM[\%] & 657 & 3.23 & 2.70 & 3.77 & 0.00 & 2.94 & 6.97 & 215.89 \\
\hline GF[\%] & 657 & 0.08 & 0.02 & 0.15 & 0.00 & 0.00 & 0.83 & 995.82 \\
\hline SG[\%] & 657 & 7.09 & 6.32 & 7.86 & 2.89 & 9.37 & 10.05 & 141.77 \\
\hline SM[\%] & 657 & 3.16 & 2.77 & 3.54 & 1.22 & 3.56 & 5.05 & 159.86 \\
\hline $\mathrm{SF}[\%]$ & 657 & 6.78 & 6.26 & 7.30 & 5.25 & 9.46 & 6.76 & 99.81 \\
\hline L_A[\%] & 657 & 62.30 & 59.86 & 64.73 & 73.78 & 85.32 & 31.76 & 50.99 \\
\hline
\end{tabular}

- Les sols de la classe A-7-5 (tableau 10) : Ils proviennent de calcaires, de shales, de Grand conglomérat, des dolomies. Ils sont constitués de $4 \%$ de graviers, de $16 \%$ de sable, $80 \%$ limons-argiles, une limite de liquidité moyenne de $50.58 \%$, un indice de plasticité moyen de $15.84 \%$, un indice de compression de 0.37 et un indice de vide moyen de 1.06. Leur indice de groupe moyen est de 14 ;

Tableau 10 : Synthèse statistique des sols A-7.5

\begin{tabular}{|c|c|c|c|c|c|c|c|c|}
\hline & $\mathbf{N}$ & Moyenne & $\begin{array}{l}\text { Int. } \\
\text { Confiance } \\
-95 \%\end{array}$ & $\begin{array}{l}\text { Int. } \\
\text { Confiance } \\
+95 \%\end{array}$ & Q1 & Q3 & $\begin{array}{l}\text { Ecart- } \\
\text { type }\end{array}$ & $\begin{array}{l}\text { Coef. } \\
\text { Var. }\end{array}$ \\
\hline $\mathrm{WI}[\%]$ & 84 & 50.58 & 49.08 & 52.07 & 48.90 & 54.00 & 6.88 & 13.61 \\
\hline Ip[\%] & 84 & 15.84 & 14.72 & 16.96 & 14.57 & 17.89 & 5.15 & 32.50 \\
\hline $\mathrm{Cc}$ & 84 & 0.37 & 0.35 & 0.38 & 0.35 & 0.40 & 0.06 & 16.96 \\
\hline e & 84 & 1.06 & 0.98 & 1.14 & 1.04 & 1.25 & 0.38 & 35.77 \\
\hline X_4.75mm[\%] & 84 & 95.28 & 92.54 & 98.01 & 99.98 & 100.00 & 12.60 & 13.23 \\
\hline X_2mm[\%] & 84 & 81.33 & 75.21 & 87.45 & 97.38 & 100.00 & 28.20 & 34.67 \\
\hline 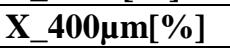 & 84 & 84.30 & 79.80 & 88.80 & 95.42 & 100.00 & 20.73 & 24.60 \\
\hline $\mathrm{X} \_80 \mu \mathrm{m}[\%]$ & 84 & 76.99 & 72.97 & 81.01 & 80.96 & 95.13 & 18.51 & 24.04 \\
\hline Ig & 84 & 14.02 & 12.59 & 15.46 & 14.00 & 19.00 & 6.62 & 47.22 \\
\hline GG[\%] & 84 & 0.00 & & & 0.00 & 0.00 & 0.00 & 0.00 \\
\hline GM[\%] & 84 & 3.53 & 2.02 & 5.04 & 0.00 & 3.49 & 6.95 & 196.74 \\
\hline GF $[\%]$ & 84 & 0.00 & & & 0.00 & 0.00 & 0.00 & 0.00 \\
\hline SG[\%] & 84 & 10.12 & 7.12 & 13.12 & 1.92 & 19.02 & 13.82 & 136.65 \\
\hline SM[\%] & 84 & 1.46 & 0.84 & 2.08 & 0.50 & 1.59 & 2.86 & 195.55 \\
\hline $\mathrm{SF}[\%]$ & 84 & 4.63 & 2.77 & 6.48 & 1.60 & 3.60 & 8.55 & 184.73 \\
\hline L_A[\%] & 84 & 62.40 & 55.14 & 69.67 & 69.24 & 90.23 & 33.48 & 53.66 \\
\hline
\end{tabular}


- Les sols de la classe A-7-6 (tableau 11) : Ils proviennent de l'altération des dolomies, des shales. Ils sont constitués de 3\% de graviers, de $14 \%$ de sable, $83 \%$ limons-argiles, une limite de liquidité moyenne de $45.73 \%$, un indice de plasticité moyen de $22.87 \%$, un indice de compression de 0.32 et un indice de vide moyen de 1.19 . Leur indice de groupe moyen est de 18 ;

Tableau 11 : Synthèse statistique des sols A-7-6

\begin{tabular}{|c|c|c|c|c|c|c|c|c|}
\hline & $\mathbf{N}$ & Moyenne & $\begin{array}{l}\text { Int. } \\
\text { Confiance } \\
-95 \%\end{array}$ & $\begin{array}{l}\text { Int. } \\
\text { Confiance } \\
+95 \%\end{array}$ & Q1 & Q3 & $\begin{array}{l}\text { Ecart- } \\
\text { type }\end{array}$ & $\begin{array}{l}\text { Coef. } \\
\text { Var. }\end{array}$ \\
\hline $\mathrm{Wl}[\%]$ & 231 & 45.73 & 44.93 & 46.53 & 43.18 & 47.91 & 6.18 & 13.50 \\
\hline Ip[\%] & 231 & 22.87 & 21.76 & 23.97 & 21.00 & 26.32 & 8.55 & 37.38 \\
\hline Cc & 231 & 0.32 & 0.31 & 0.33 & 0.30 & 0.34 & 0.06 & 17.28 \\
\hline e & 231 & 1.19 & 1.13 & 1.26 & 1.10 & 1.33 & 0.49 & 41.00 \\
\hline $\mathrm{X}_{\mathrm{C}}^{4.75 \mathrm{~mm}[\%]}$ & 231 & 94.43 & 92.24 & 96.62 & 100.00 & 100.00 & 16.92 & 17.92 \\
\hline $\mathbf{X} \_2 \mathrm{~mm}[\%]$ & 231 & 88.30 & 85.26 & 91.35 & 99.43 & 100.00 & 23.48 & 26.59 \\
\hline $\mathrm{X}_{-400 \mu \mathrm{m}[\%]}$ & 231 & 86.76 & 84.01 & 89.51 & 95.91 & 100.00 & 21.21 & 24.45 \\
\hline $\mathrm{X}_{-} 80 \mu \mathrm{m}[\%]$ & 231 & 77.55 & 75.38 & 79.73 & 80.60 & 92.03 & 16.79 & 21.65 \\
\hline Ig & 231 & 17.70 & 16.60 & 18.81 & 18.00 & 22.00 & 8.53 & 48.19 \\
\hline GG[\%] & 231 & 0.00 & 0.00 & 0.01 & 0.00 & 0.00 & 0.03 & 1519.87 \\
\hline GM[\%] & 231 & 2.87 & 2.00 & 3.74 & 0.00 & 2.06 & 6.73 & 234.62 \\
\hline GF[\%] & 231 & 0.10 & 0.00 & 0.20 & 0.00 & 0.00 & 0.78 & 773.01 \\
\hline SG[\%] & 231 & 6.38 & 5.01 & 7.76 & 1.65 & 7.79 & 10.63 & 166.42 \\
\hline SM[\%] & 231 & 2.01 & 1.49 & 2.52 & 0.63 & 2.08 & 3.98 & 198.18 \\
\hline SF[\%] & 231 & 5.50 & 4.47 & 6.53 & 3.39 & 6.73 & 7.92 & 144.02 \\
\hline$L_{-} \mathbf{A}[\%]$ & 231 & 55.86 & 51.06 & 60.67 & 69.24 & 87.70 & 37.05 & 66.33 \\
\hline
\end{tabular}

La figure 3 donne une synthèse de la distribution granulométrique des différentes classes des sols identifiés.

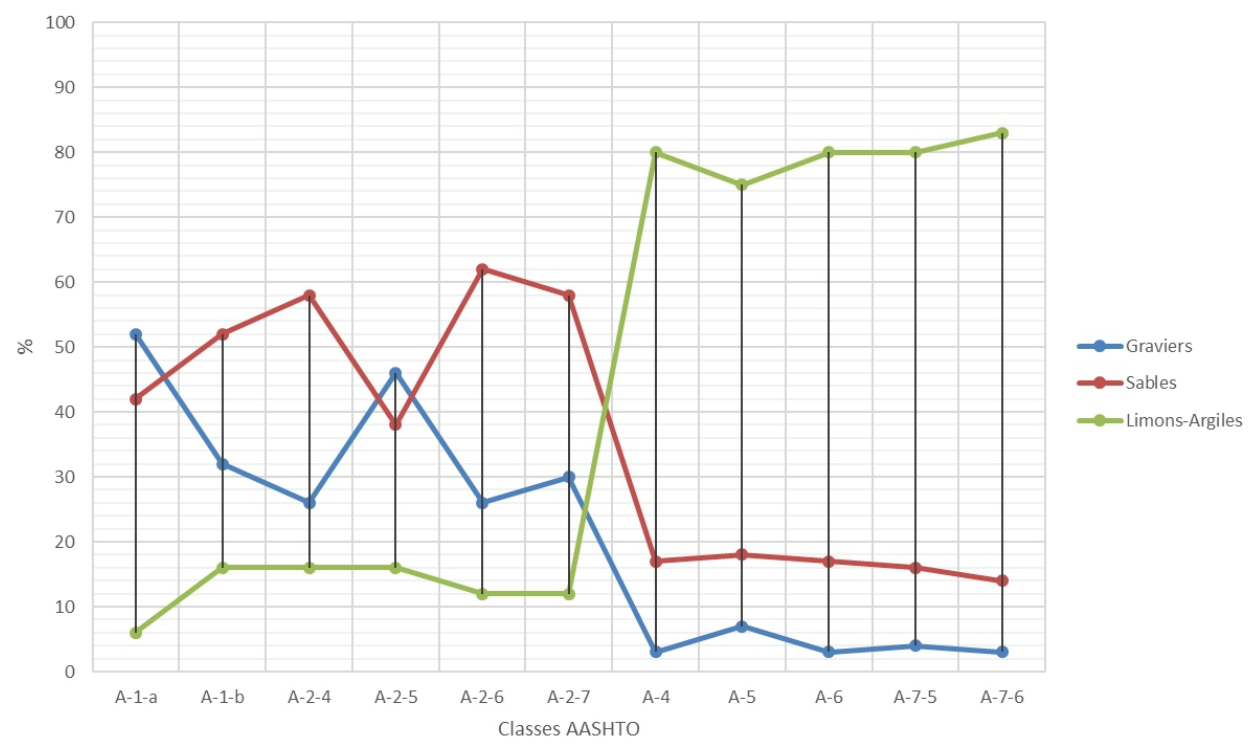

Figure 3 : Distribution granulométrique dans différentes classes AASHTO 
La distribution spatiale de chacune de classe géotechnique est présentée sur la carte de la figure 4. Pour la lisibilité de l'information cartographique, certains secteurs de la figure 4 ont été agrandis tels que présenté sur les figures 5, 6 et 7 .

- Les sols constituent
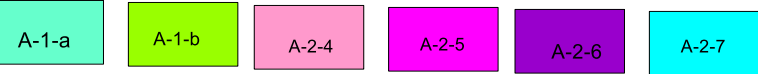
des bons supports des fondations. Ces sols constituent des sites favorables aux cimétières (sols aérés). Ils constituent également des aquifères potentiels. Ils correspondent insitu respectivement à des : graviers sablonneux à limons et argiles, sables graveleux à limons et argiles, sables graveleux à limons, graviers sablonneux à limons plastiques, sables graveleux à argiles et sables graveleux à argiles plastiques. Les sols latéritiques font parti de ces classes. A Lubumbashi, ces sols sont autochtones ;

- Les sols A-4 $_{\text {A-5 }}$ sont des limons pulvérulents et plastiques à sables. Ce sont des bons supports fondations, mais très vulnérables à l'érosion hydrique (surtout la classe A-4). Comme les sols décrits précédemment, ils sont autochtones ;

- Les sols A-6 A-7-5 A-7-6 sont argileux à sables. Les sols A-7 constituent de très mauvais supports des fondations à cause de leur plasticité élévée. Ces sols constituent des sites favorables aux zones de stockage des dechets (capacité de rétention spécifique des argiles très élévée par rapport aux autres minéraux contitutifs des sols). A Lubumbashi ces sols sont autochnones ; 


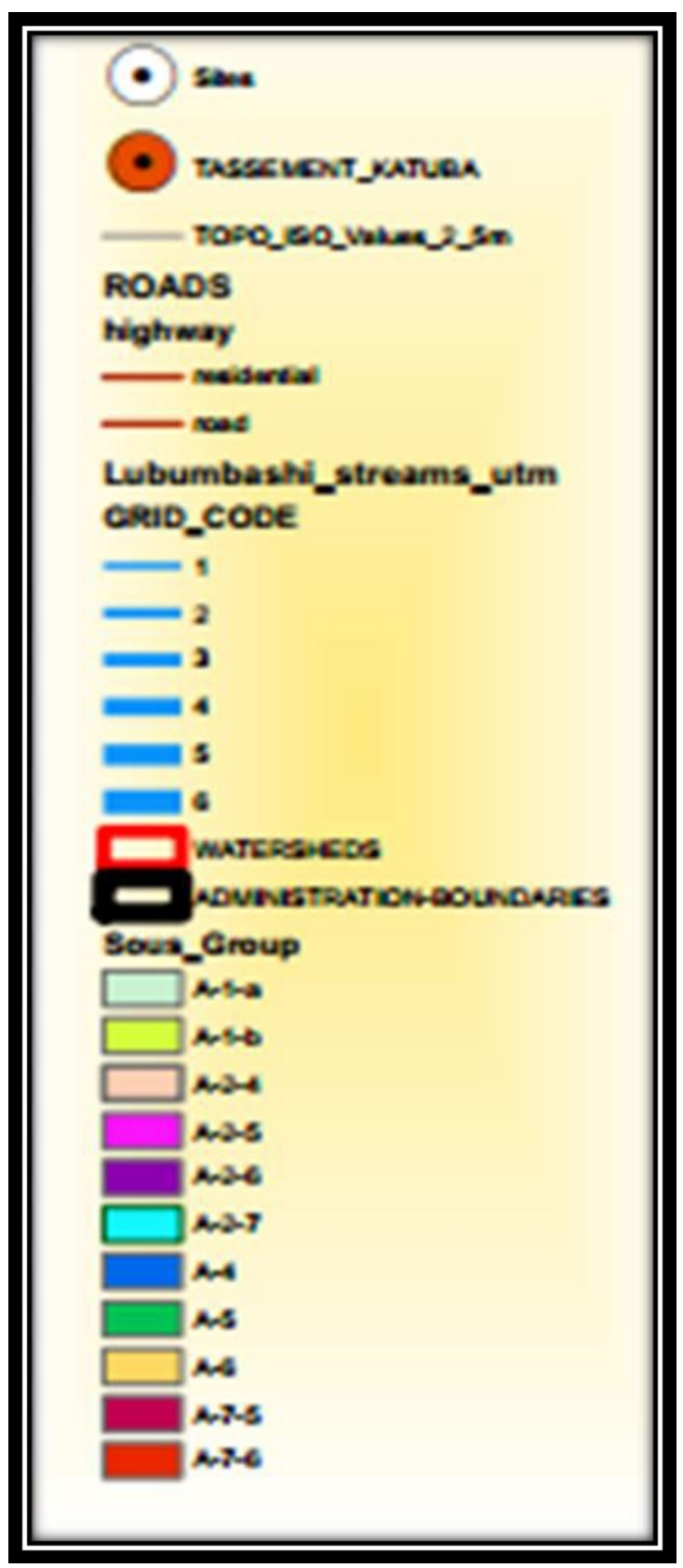




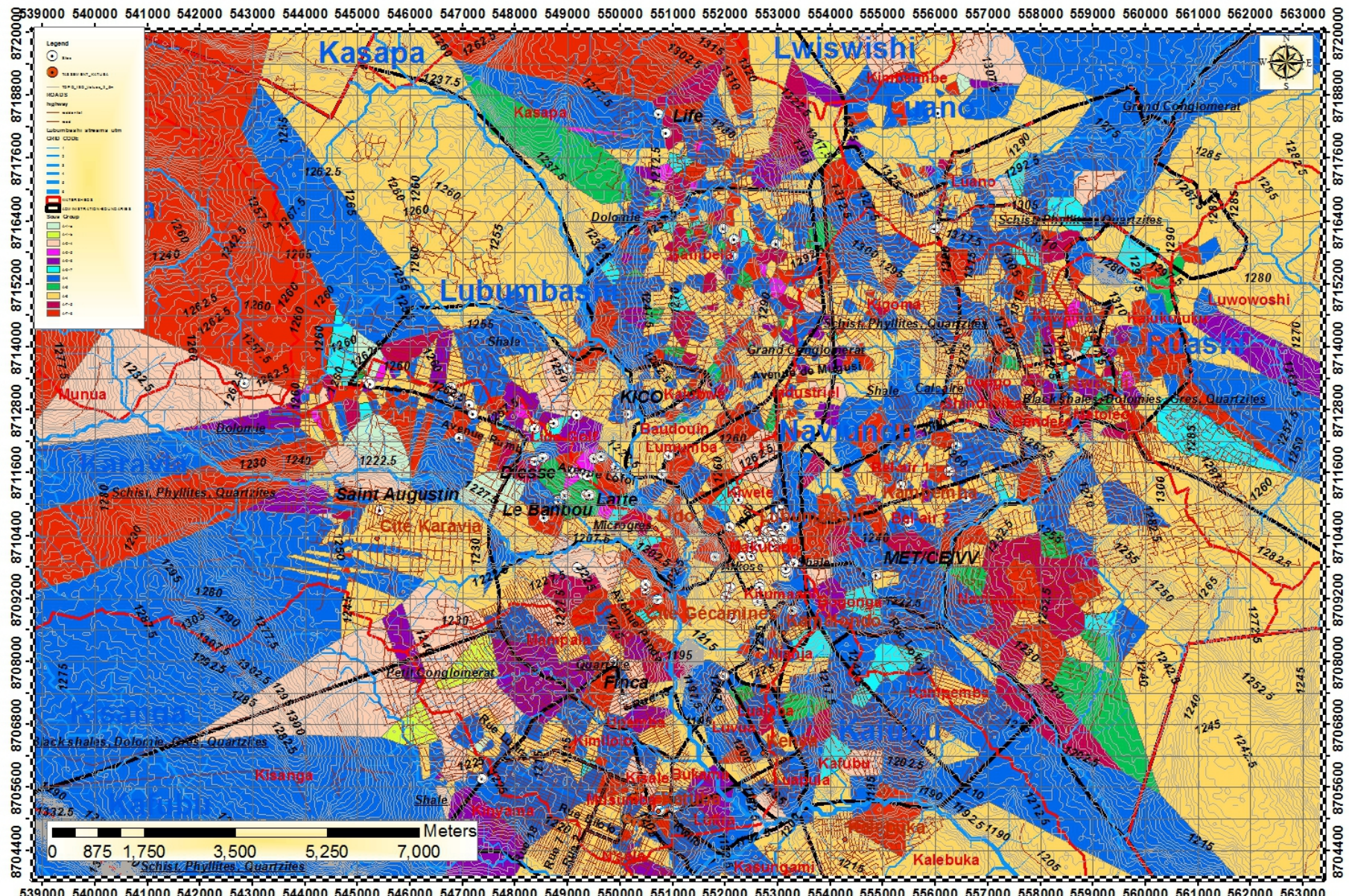

Figure 4: Carte géotechnique d'ensemble de la ville de Lubumbashi 


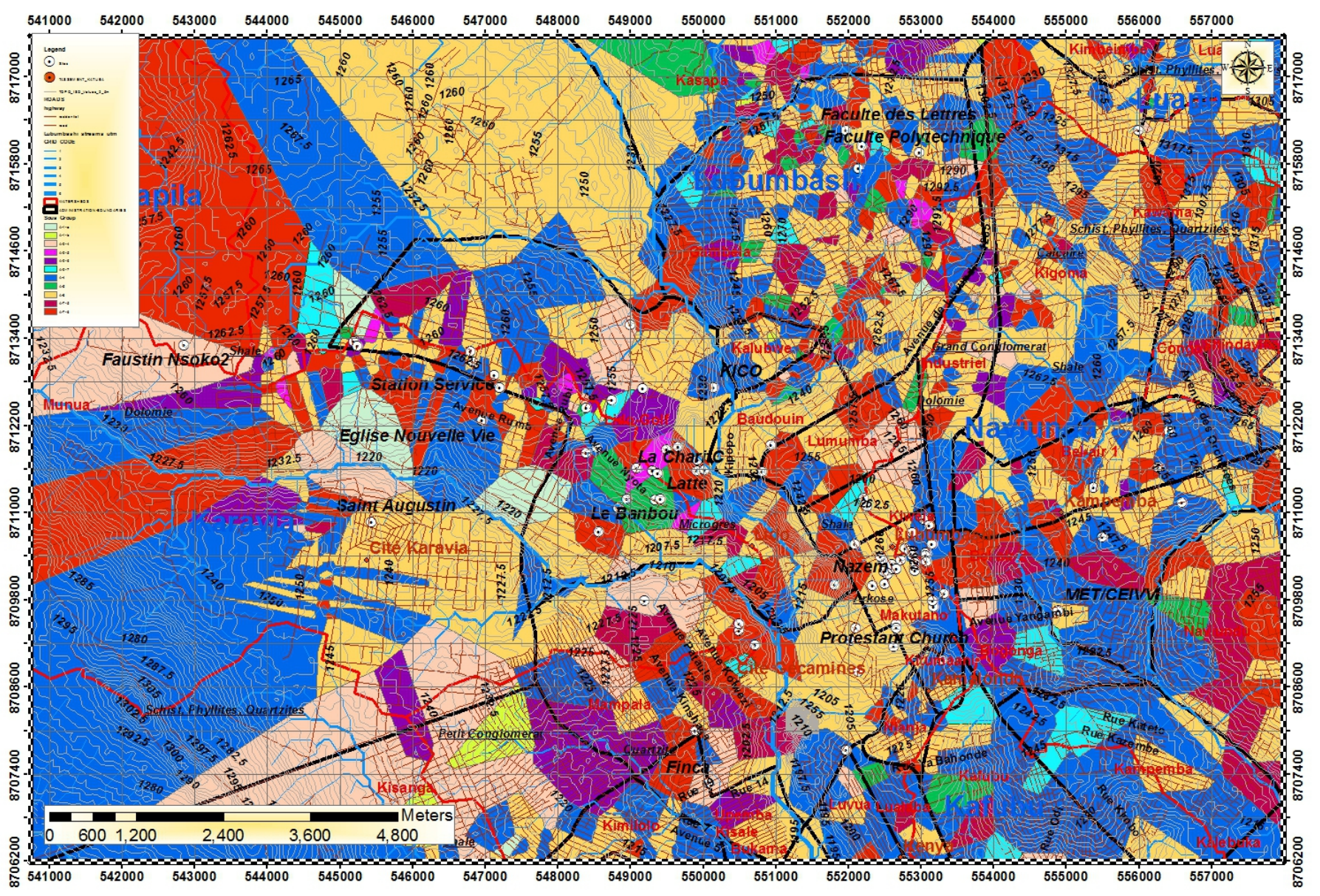

Figure 5: Carte géotechnique de la partie centrale de la ville de Lubumbashi 


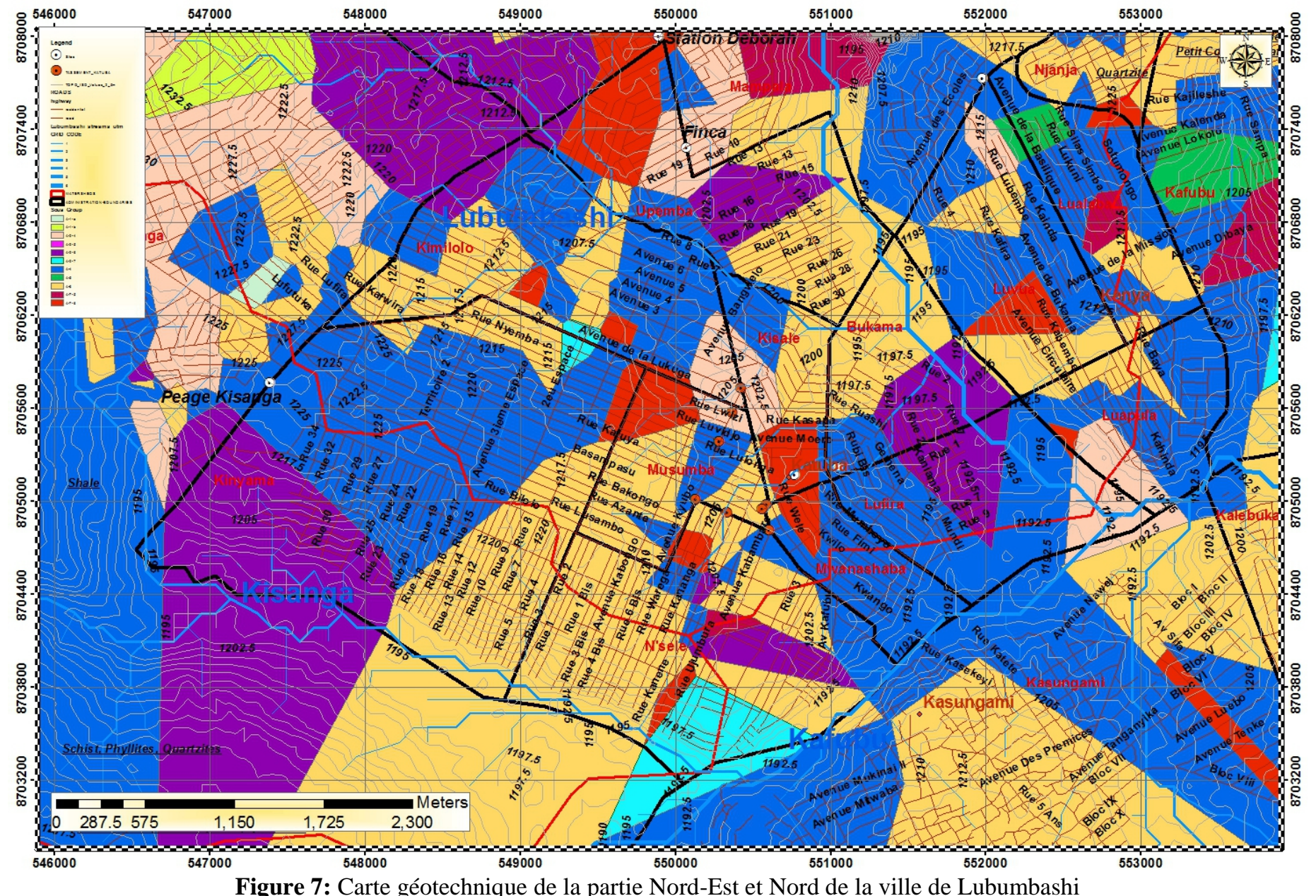




\section{Conclusion}

Du point de vue géotechnique, les sols de Lubumbashi se répartissent en 11 sous-groupe AASHTO: A-1-a (graviers sablonneux à argiles), A-1-b (sables graveleux à argiles), A-2-4 (sables graveleux à limons), A-2-5 (graviers sablonneux à limons plastiques), A-2-6 (sables graveleux à argiles), A-2-7 (sables graveleux à argiles actives), A-4 (limons à sables), A-5 (limons plastiques à sables), A-6 (argiles à sables), A-7-5 (argiles actives à sables), A7-6 (argiles actives à sables). Les sols à indice 5 contiennent des argiles de type illites tandisque les autres sont constitués des kaolinites sur l'abaque de plasticité de Casagrande.

En tenant compte de l'indice de groupe, ces sols sont répartis en 130 classes AASHTO:

- La classe modale correspond aux sols A-6 caractérisés par des indices de groupe allant de 0 à 22 ;

- Les sous-groupes A-1-a, A-1-b, A-2-4, A-2-5, A-2-6 et A-2-7 sont caractérisés par un indice de groupe de 0 ;

- Les sous-groupes A-4 et A-5 sont caractérisés par des indices de groupe variant de 0 (mode) à 9 ;

- Les sous-groupes A-7-5 et A-7-6 présentent des matériaux à indice de groupe élevé variant entre 10 et 26 avec 18 comme valeur modale.

Les sols de classe A-1 et A-2, constituent des bons supports de fondations d'ouvrages et forment les aquifères potentiels de la ville de Lubumbashi (nappe d'eau souterraine, zone de drainage, zone d'alimentation des nappes). Ils constituent également des sites favorables à l'implantation des cimétières (sols aérés) car le délai nécessaire à la destruction de corps est bref. Dans ce cas la rotation de 5 ans peut être utilisée sans problème pour une ville à population dense comme la ville de Lubumbashi.

Les sols A-4 et A-5 constituent également des bons sous-bassement des fondations, mais sont très vulnérables à l'érosion et à la liquefaction.

Les sols A-6 et A-7 forment des substratums imperméables des aquifères. Ces sites peuvent servir à la mise en place des centres d'enfouissement technique. Ils sont également considérés comme des gisements d'argiles dans la fabrication des ceramiques et des produits refractaires.

En tenant compte de la nature des roches mères, les sols A-1 et A-2 proviennent des conglomérats, des grès et du processus de latéritisation des formations rocheuses très riches en fer comme les shales. Les sols A-4 et A-5 sont souvent des produits d'altération des shales gréseux et des microgrès tandis que les sols A-6 et A-7 proviennent de l'altération des roches argileuses et dolomitiques : les shales, les shales gréseux, les calcaires et les dolomies. 
La carte géologique est un document nécessaire mais non suffisante pour les études géotechniques. Une étude détaillée des sols est et l'état d'altération des différentes parties d'un massif rocheux est inévitable.

Cet article est un document guide qui facilitera d'autres investigations comme le mesurage des caractéristiques de déformabilité et de cisaillement des sols, les essais pénétrométriques. Ceci permettra d'évaluer des contraintes admissibles et d'estimer des tassements anticipés des sites d'érection d'ouvrages en fonction de la stratigraphie de chaque site.

\section{References:}

1. Addinsoft 2007. Addinsoft, http://www.addinsoft.com, 596p.

2. Aeg, Saice, et Saieg 2001. Guidelines for soil and rock logging in South Africa. A.B.A. Brink et R.M.H. Bruin, 47p.

3. E. Bagarre 1990. Utilisation des graveleux latéritiques en techniques routières. ISTED, 38, rue Liancourt-75014 Paris (France), 148p.

4. W. Christopher et C. Susan 2001. Spatial statistics in crime analysis: using CrimeStat III. Natianal Institute of Justice (NIJ), 2.1 edition, $151 \mathrm{p}$.

5. J. Costet et G. Sanglerat 1975. Cours pratique de mécanique des sols, plasticité et calcul des tassements, volume Tome 1. Dunod, 2ème edition, 262p.

6. M. DAS et K. Sobhan 2012. Principles of geotechnical engineeting. SI, viii edition, 2012. 770p.

7. P. De-Clerck 1977. Limites d'Atterberg, rapport d'un étalonnage croisé et d'essais complémentaires. Rapport, C.R.R, Belgique, 82p.

8. X. Dupre 2010. Langages Informatiques, Programmation avec le langage Python, Un outil commode au service de l'ingénieur. ENSAE, 336p.

9. M. Dysli 2001. Recherche bibliographique et synthèse des corrélations entre les caractéristiques des sols. Rapport 314, Département fédéral de l'environnement, des transports, de l'énergie et de la communication, Office fédéral des routes, Laboratoire de mécanique des sols de l'EPFL-Suisse, 314p.

10. J. Edzer S. Roger et G. Virgilio 2008. Applied spatial data analysis with $R$. Springer, 378p.

11. J. Hunter, D. Dale, E. Firing, et M. Droettboom 2016. Matplotlib. release $1.5 .1,2864 \mathrm{p}$.

12. P. Kasongo 2017. Mise en place d'un système d'informations géotechniques, validation avec des données géotechniques de la ville de lubumbashi en République Démocratique du congo. Master's thesis, Université de Lubumbashi, Lubumbashi, 155p. 
13. P. Laffitte 1972. Traité d'informatique géologique. Masson et Cie, $624 \mathrm{p}$.

14. G. Lorenzo 2006. Analyse d'inégalité, l'indice de gini. FAO, www.fao.org/tc/easypol, 30p.

15. E. Lousberg 1978. Mécanique des sols, essais de chantier. Rapport, Université Catholique de Louvain, Belgique, 90p.

16. M. Massamba 2013. Contribution à l'étude de sols latéritiques du Sénégal et du Brésil. PhD thesis, Université Cheikh Anta Diop DE Dakar, Dakar, 163p.

17. W. Mckinney et PyData Development Team 2014. Pandas: powerful python data analysis toolkit.release 0.15.1. http://github.com/pydata/pandas, $1757 \mathrm{p}$.

18. PySAL-Developers 2015. Pysal documentation. release 1.11.0dev, $503 \mathrm{p}$.

19. N. Rappin et R. Dunn 2006. wxPython in action. Manning, 583p.

20. G. Sanglerat et B.C. Olivari 1983. Problèmes pratiques de mécanique des sols, Généralités, plasticité, calcul des tassements, Interprétation des essais in situ, Tome 1, Dunod, 327p.

21. SciPy-community 2015. Scipy reference guide. release $0.7 . d e v, 435 \mathrm{p}$.

22. Sonia, Nkulu, Kipata, Lubala, Lunda 2013: l'Urbanisation et l'environnement géologique de la ville de Lubumbashi (Katanga, R.D.Congo), Presses Universitaires, Unilu.

23. Usda 1987. Soil mechanics level I, module 2: Aashto (American Association of State Highway and Transportation Officials), study guide. Soil Concervative Service, 71p.

24. J. Verdeyen, V. Roisin et J. Nuyens 1968. La mécanique des sols, volume Tome 2. Dunod, 508p 